\title{
Activation of MAP kinase pathways in Galleria mellonella infected with Bacillus thuringiensis
}

\author{
Iwona Wojda ${ }^{凶}$, Konrad Koperwas and Teresa Jakubowicz \\ Department of Immunobiology, Institute of Biology and Biochemistry, Faculty of Biology and Biotechnology, Lublin, Poland
}

\begin{abstract}
We followed changes in the level of phospho-MAP kinases in the greater wax moth Galleria mellonella after infection with Bacillus thuringiensis. We observed an enhanced level of phosphorylated p38 and JNK in fat bodies of the infected larvae. In hemocytes, injection of $B$. thuringiensis caused the highest increase in phosphoJNK, however, all pathways were activated after aseptic injection. We report that Galleria mellonella larvae exposed to heat shock before infection showed an enhanced level of phosphorylated JNK in fat body. This finding is relevant in the light of our previous reports, which submit evidence that pre-shocked animals are more resistant to infection.
\end{abstract}

Key words: Bacillus thuringiensis, Galleria mellonella, heat shock, infection, innate immunity, MAP kinases

Received: 24 October, 2013; revised: 09 January, 2014; accepted: 20 January, 2014; available on-line: 22 January, 2014

\section{INTRODUCTION}

The MAP kinase pathways are important regulators of cellular physiology in all eukaryotic organisms. They consist of the evolutionary well-conserved „core" created by 3 kinases: MAPKKK (MAP kinase kinase kinase) which after receiving a signal activates the MAPKK (MAP kinase kinase), that in turn regulates the activity of MAPK (MAP kinase). The last MAPKs phosphorylate a range of protein substrates including downstream signalling proteins, diverse enzymes, and cytoskeletal proteins. Among proteins regulated by signal- transduction pathways there are also transcription factors controlling the expression of signal-specific genes. The main MAP kinase pathways are named after their MAP kinase: ERK, p38, and JNK. ERK pathway, whose activation leads to phosphorylation of ERK in threonine and tyrosine residues in the conservative motif T-E-Y, is activated mostly by growth factors and mitogens. The other two pathways possess p38 and JNK MAP kinases phosphorylable in the motive T-G-Y and T-P-Y, respectively, and are mostly activated under stress conditions such as elevated temperature, oxidative and osmotic stress, etc. (Boutros et al., 2008). ERK subgroup of MAP kinases seems to be phylogenetically the oldest, while SAP kinases (stressactivated protein kinases): p38 and JNK appeared later in evolution in animals and fungi, but they are absent in plants. It is worth mentioning that there are multiple forms of each MAPK subtype in different organisms. For example, in mammals two forms of ERK were found (ERK1 and ERK2), and phylogenetically distant BMK1/ERK5, four p38 (p38 $\alpha$, p38 $\beta$, p38, p $38 \gamma$ ), and three JNK (JNK1, JNK2, JNK3). The budding yeast Saccharomyces cerevisiae uses at least 5 MAP cascades responding to mating pheromones, limitation of nitrogen, growth factors and cell-wall damage, osmotic stress, and starvation (Wojda, 2010, 2012).

The existence of MAP kinase pathways allows cells to perceive and amplify signals and adjust their metabolism to different environmental factors, both abiotic and biotic. It is known that MAP kinases regulate immune response in mammals (Zhang \& Dong, 2005). Much less is known about their role in the defence system in insects that lack the adaptive immunity and rely only on innate reactions. Taking into account that immune response is well evolutionarily conserved, the usage of insect models may be very useful in understanding the mechanism of innate immunity. It is worth mentioning that the discovery of Toll receptors in Drosophila melanogaster had a huge impact on the discovery of infection recognition in mammals, which was awarded the Nobel Prize in 2011.

Unlike mammals, insects do not possess T-cells, Bcells, or antibodies. Their immune system is simpler and comprises humoral and cellular events. In the bestknown insect model Drosophila melanogaster, the humoral response is mediated inter alia by Toll and Imd path-

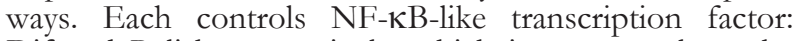
Dif and Relish, respectively, which in turn regulates the expression of genes encoding antimicrobial peptides (Lemaitre et al., 1995; 1996; Khush et al., 2001). These defence peptides secreted by insect fat body and other tissues into the hemolymph act against infecting microorganisms, mostly by destroying the cell membranes ( $\mathrm{Li}$ et al., 2012). Also as a part of humoral immune response, clotting and melanisation processes are involved. Cellular response engages insect blood cells - hemocytes, which are able to phagocytose microorganisms. Many infecting microorganisms or larger pathogens may be sequestered from the host organisms closed in specific hemocytebased structures called nodules or capsules (Williams, 2007).

In this report, we analyse the activation of MAP kinase pathways in fat body and hemocytes of the greater wax moth Galleria mellonella infected with enthomopathogenic bacteria Bacillus thuringiensis. This microorganism is used for production of bioinsecticides. In addition, genes encoding toxins produced by Bacillus are introduced into the plant genome to increase resistance to pests. Galleria mellonella is an insect model commonly used to study the biochemical aspects of innate immunity due to the fact,

e-mail: wojda@hektor.umcs.lublin.pl

Abbreviations: Aminoacids: T - threonine, $Y$ - tyrosine, G - glycine, $\mathrm{E}-$ glutamic acid, $\mathrm{P}$ - proline, $\mathrm{X}$ - any aminoacid; MAP kinase (Mitogen Activated Protein Kinase), SAP kinases - Stress Activated Protein Kinases 
that contrary to D. melanogaster (which is used to investigate the genetic aspects of immunity) the larvae are big enough to isolate hemolymph easily, as well as fat body, or other immune-relevant tissues/organs (Glavis-Bloom, 2012). Greater wax moth caterpillars feed on honeycomb inside bee nests and therefore they are considered as pests.

\section{MATERIALS AND METHODS}

Insects, bacteria, and infection. Galleria mellonella (Lepidoptera: Pyralidae) were reared on honeybee nests debris at $28^{\circ} \mathrm{C}$ in darkness. Last instar larvae of about $200 \mathrm{mg}$ were used for the infection experiments. Bacillus thuringiensis (B.t. kurstaki HD1, Scientific Research Institute of Plant Protection Methods, Kishinev, Moldova) were grown in LB medium (1\% bactotryptone, $1 \%$ $\mathrm{NaCl}, 0.5 \%$ yeast extract) at $37^{\circ} \mathrm{C}$ with shaking. Afterwards, the culture was centrifuged and suspended in IPS buffer $(150 \mathrm{mM} \mathrm{NaCl}, 5 \mathrm{mM} \mathrm{KCl}, 100 \mathrm{mM}$ Tris- $\mathrm{HCl}$ $\mathrm{pH}$ 6.9) to the respective density $\left(4 \times 10^{3}\right.$ cells in $\left.5 \mu \mathrm{l}\right)$ and injected into larval hemocel using a Hamilton syringe. Control larvae were injected with IPS alone.

Isolation of $G$. mellonella fat bodies and hemocytes. The caterpillars were anesthetized by cooling down in cold water and surface sterilized in 70\% ethanol. The fat bodies were isolated under cell-culture grade, ice-cold Ringer's solution (172 mM KCl, $68 \mathrm{mM} \mathrm{NaCl}$, $5 \mathrm{mM} \mathrm{NaHCO}$, pH 6.1, osmolarity $420 \mathrm{mOsm})$. The organs were transferred to Eppendorf tubes containing Ringer's solution. The liquid was removed and fat bodies were quickly frozen in liquid nitrogen. They were kept at $-20^{\circ} \mathrm{C}$ for further use. The fat bodies from 5 larvae were used for each time-point.

In order to isolate hemocytes, Galleria mellonella larvae were cooled down, washed in apyrogenic water, and sterilized in $70 \%$ ethanol. Larvae were injured with a sterile needle, and 10-40 $\mu$ l of hemolymph was added to $500 \mu \mathrm{l}$ of ice-cold IPS. The hemocytes were sedimented by centrifugation $200 \times g$ for 5 minutes at $4^{\circ} \mathrm{C}$, washed with IPS to get rid of the remaining hemolymph components, and frozen in liquid nitrogen. They were kept
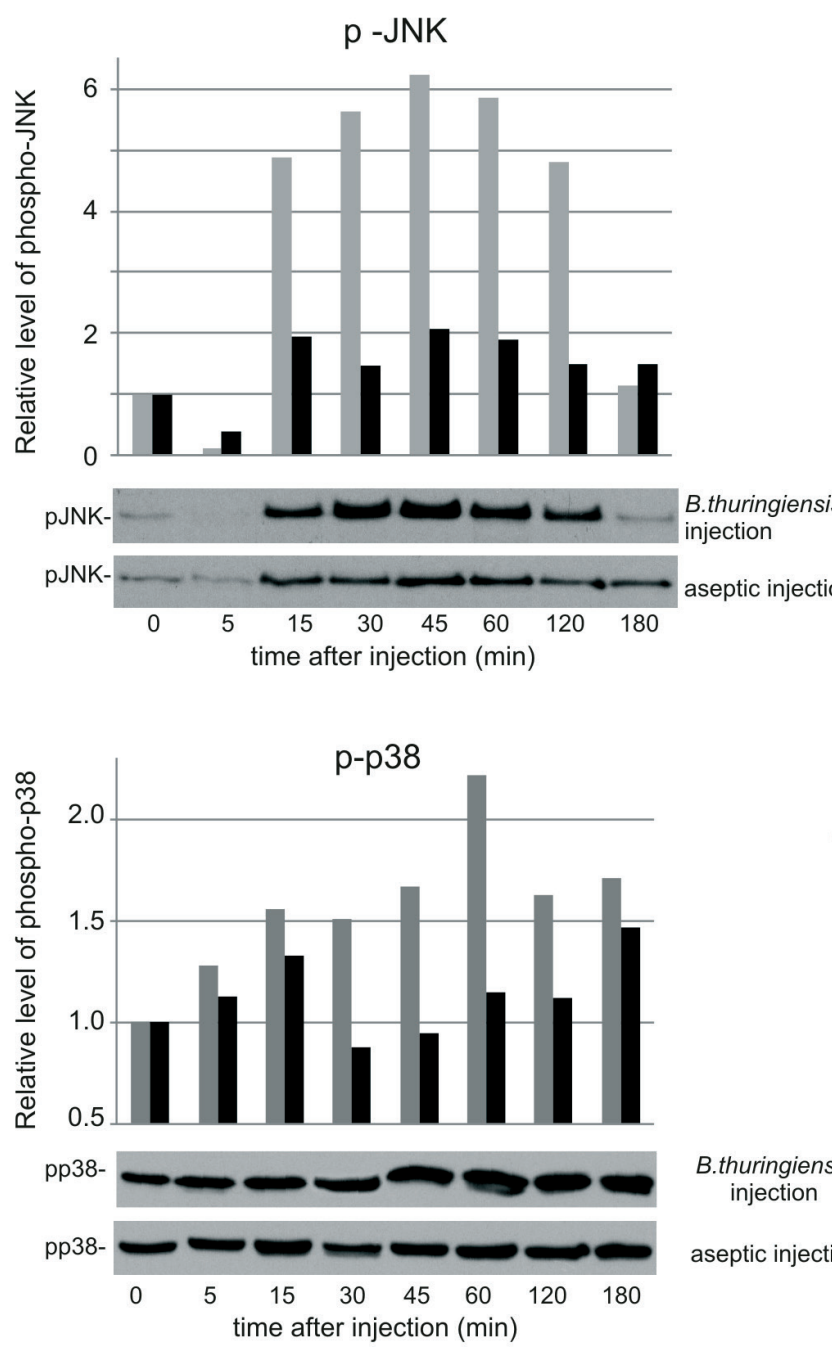

Bacillus thuringiensis injection

aseptic injection

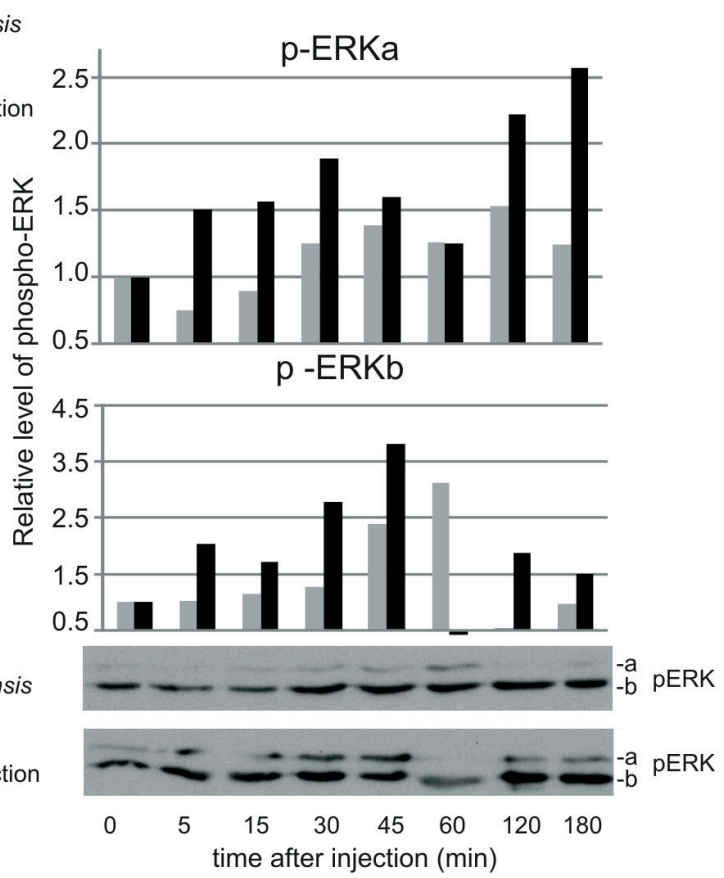

Figure 1. Detection of phospho-MAP kinases in G. mellonella fat body.

The corresponding phospho-MAP kinase (p-p38, p-ERK and p-JNK) was detected after aseptic injection with IPS (shown as lower panels with the relative quantification depicted by black bars) and after injection of $B$. thuringiensis cells (upper panels, depicted quantitatively by grey bars). For the quantitative analysis the amount of each phospho-protein in time 0 (intact larvae) was taken as a reference sample. Twenty micrograms of protein extracts from fat bodies isolated at the indicated time-points after injection were immunoblotted with the respective anti-phospho MAP antibodies (p-JNK, p-p38, p-ERK). A representative experiment is shown. 
at $-20^{\circ} \mathrm{C}$ for further use. Hemolymph from 8-10 larvae was used for each time-point.

Preparation of protein extracts and western-immunoblotting. Frozen fat bodies or hemocytes, prepared as described above, were thawed on ice and lysed with buffer: $50 \mathrm{mM}$ Tris- $\mathrm{HCl} \mathrm{pH} 7.5,1 \%$ deoxycholate sodium salt, 1\% Triton X-100, 0.1\% SDS, 50 $\mathrm{mM} \mathrm{NaF}, 5 \mathrm{mM} \mathrm{Na}$ pyrophosphate, $0.1 \mathrm{mM}$ sodium orthovanadate, $1 \mathrm{mM}$ PMSF, and $\alpha$ complete (Roche, $400 \mu \mathrm{l}$ per $10 \mathrm{~mL}$ of buffer). They were disintegrated using a pellet pestle mixer (Roche) and centrifuged for $15 \mathrm{~min}$ at $10000 \times g$ to remove cell debris. The entire procedure was performed at $4^{\circ} \mathrm{C}$. Protein concentration was estimated according to Bradford (1976). Samples were resolved on $10 \%$ SDS polyacrylamide gel according to Laemmli (1970). After the run, gels were electroblotted onto PVDF membranes for 1.5 hour at $350 \mathrm{~mA}$. Blots were probed with appropriate primary anti-phospho MAPK (1:1000) antibodies, and then with horseradish-peroxidase-conjugated goat anti-rabbit IgG (1:2000). Immune complexes were visualized using an enhanced chemiluminescence system (New England, Biolabs) according to the manufacturer's specification. A video image analyser Chemi Doc MP ImagingSystem

Bacillus thuringiensis injection

aseptic injection

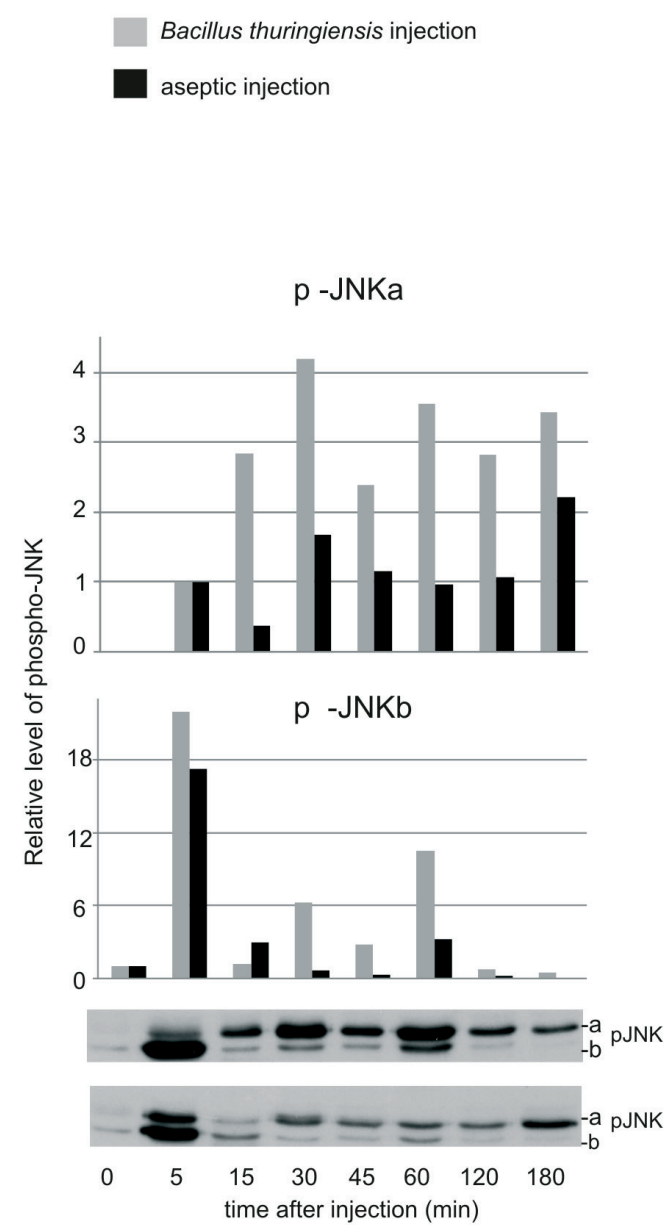

(Bio Rad) was used for densitometric analysis of the obtained signal.

\section{RESULTS}

Taking advantage of the fact that MAP kinases are highly conserved from yeast to mammals, we used antibodies directed against dually phosphorylated and hence activated forms of p38, ERK, and JNK kinases to study the phosphorylation level of MAP kinases in G. mellonella larvae. These antibodies recognised the respective enzymes only in their dually phosphorylated forms (at threonine and tyrosine residue separated by the respective aminoacid characteristic for each type of kinase) in their activation loop. They recognised proteins of molecular weight corresponding to the weight of MAP kinases (all between $37 \mathrm{kDa}$ and $50 \mathrm{kDa}$, not shown).

We noticed that aseptic injection caused an increase in the amount of phosphorylated forms of all MAP kinases in the insect fat bodies. Nevertheless, the level of phosphorylated forms of stress-activated protein kinases (SAPK): JNK and p38 was significantly higher when $\mathrm{Ba}$ cillus thuringiensis cells were injected into larval hemocel. In the case of JNK, the enhanced phosphorylation was

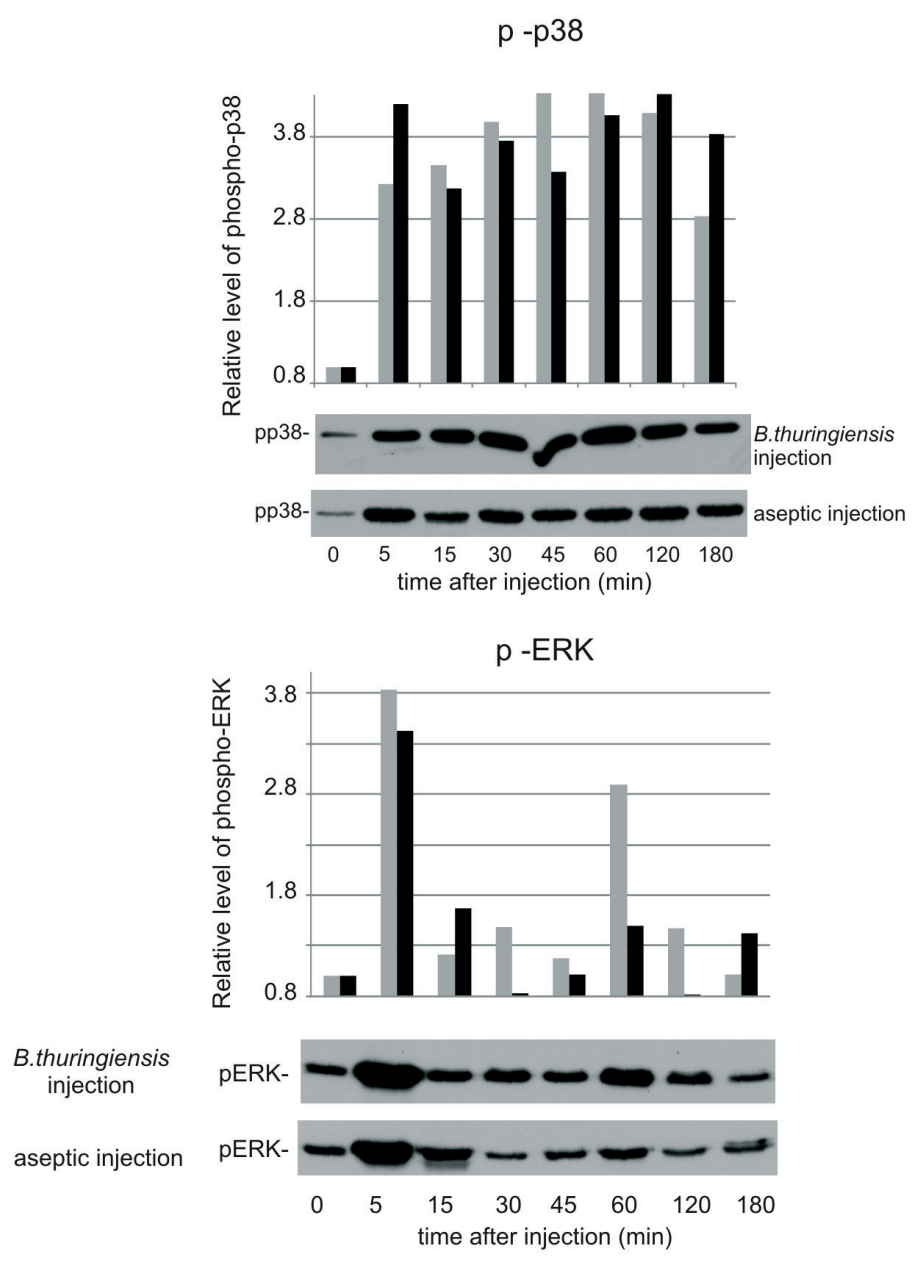

Figure 2. Detection of phospho-MAP kinases in G. mellonella hemocytes.

The corresponding phospho-MAP kinase ( $p$-p38, p-ERK and p-JNK) was detected after aseptic injection with IPS (shown as lower panels with the relative quantification depicted by black bars) and after injection of $B$. thuringiensis cells (upper panels, depicted quantitatively by grey bars). Protein extracts $(20 \mu \mathrm{g})$ from hemocytes were immunoblotted with the respective anti-phospho MAP antibodies (p-JNK, $\mathrm{p}$-p38, p-ERK). For the quantitative analysis the amount of phospho-protein in time 0 (intact larvae) was taken as a reference sample in the case of p38, ERK and JNKb. Since no phospho-JNKa was detected in naïve larvae (time 0), the sample from the 5-minute time-point was taken as a reference sample. A representative experiment is shown. 


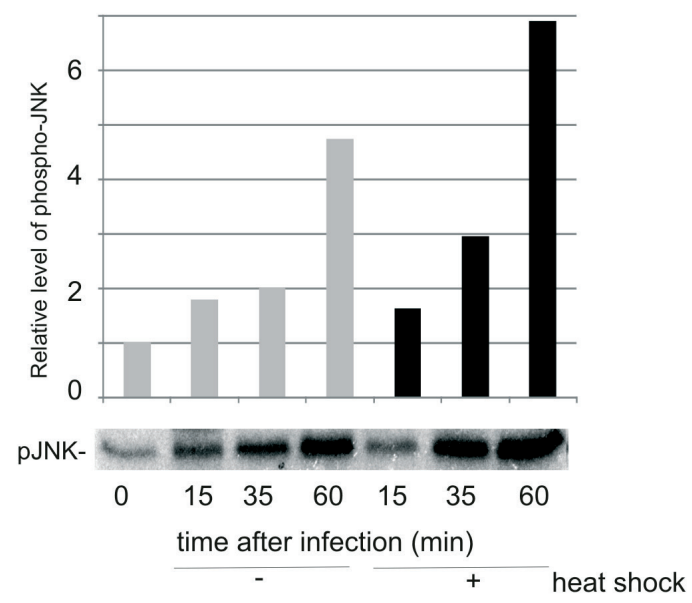

Figure 3. Detection of phospho-JNK MAP kinase in fat bodies of G. mellonella larvae exposed $(+)$ or not exposed $(-)$ to heat shock $\left(40^{\circ} \mathrm{C}, 30 \mathrm{~min}\right)$ directly before infection with $B$. thuringiensis.

After infection, all larvae were reared at $28^{\circ} \mathrm{C}$. At the indicated time-points after infection, fat bodies were isolated, protein extracts were prepared, and detection was performed as described in the Material and Methods Section. A representative experiment is shown.

observed 15-120 minutes after infection, whereas Bacillus-dependent p38 activation was detected later: 30-180 minutes after infection (Fig. 1 - left part). Likewise in our previous report (Wojda et al., 2004), two forms of phospho-ERK were recognised ( $\mathrm{a}$ and $\mathrm{b}$ ). We noticed an increased level in their amount after aseptic injection itself, but no significant Bacillus-specific phosphorylation was detected (Fig. 1 - right part).

We also followed the phosphorylation status of MAP kinases in the hemocytes of the infected $G$. mellonella larvae. In contrast to the fat body, one band corresponding to the molecular weight of MAP kinases was detected by anti-ERK antibodies and two bands by anti-JNK antibodies ( $\mathrm{a}$ and $\mathrm{b}$ ). Injection of $B$. thuringiensis cells caused a slight (p38) or clear (ERK) increase in the amount of phosphorylated forms of the respective MAPK (Fig. 2 - right part) in comparison to the aseptic injection. However, the clearest Bacillusdependent increase in the amount of phospho-protein was detected for JNK MAP kinase (JNKa). This form of MAP kinase was not detected in intact larvae. The other form of phospho-JNK (JNKb) was detectable in intact larvae, slightly increased in IPS injected animals, but the highest level of this phospho-protein was observed after B. thuringiensis injection (Fig. 2 - left part). It is worth noting that all MAP kinases were phosphorylated quickly and strongly after aseptic injection itself (IPS injection only). Phospho-p38 remained at the enhanced level at all time-points tested, while activation of ERK appeared to be more transient.

Because of our previous observations concerning the positive effect of heat shock on insect's resistance, we decided to compare the phosphorylation level of JNK MAP kinase in fat bodies of $G$. mellonella larvae preexposed or not to heat shock directly before injection of B. thuringiensis cells. Interestingly, we noticed that in shocked G. mellonella larvae, the phosphorylation level of JNK MAP kinase was significantly higher than in non-shocked animals (at 35 and 60 minutes after infection). These results may bring us closer to understanding the biochemical causes of increased resistance in heatshocked animals.

\section{DISCUSSION}

Living organisms are continuously exposed to various environmental stresses such as too high or too low temperature, ultraviolet radiation, oxidants, free radicals, and infection. In order to respond appropriately and to maintain organism's integrity, they evolved intracellular signalling pathways. These include MAP modules, which are relatively well known in human and yeast cells, although still the mechanisms by which they are regulated are not fully discovered. In the best-known insect model, the fruit fly Drosophila melanogaster, genes encoding components of MAP pathway modules have been found. These encoding ERK and JNK are named rolled and basked, respectively. Genes encoding p38: $p 38 a$, p38b and p38c have also been identified (www.flybase.org). The genome of Galleria mellonella is not the end of outdoor and the genes encoding MAP kinases have not been cloned. By the use of specific antibodies recognising dually phosphorylated forms of p38, JNK and ERK, we were able to follow their level in the fat body and hemocytes of the infected G. mellonella larvae. The fat body is a dynamic organ unique for insects and involved in many metabolic functions. It controls the synthesis and utilization of energy reserves such as glycogen and lipids. Also, most of the circulating hemolymph proteins and peptides are synthesized in this organ including defence peptides appearing in the hemolymph as part of the humoral immune response (Arrese \& Soulages, 2010). We report that p38 and JNK stress pathways are highly activated in fat body in response to infection with the enthomopathogenic bacterium Bacillus thuringiensis. JNK activation was transient, noticeable 15 minutes after injection of bacteria, and lasted for about 2 hours. This is consistent with our earlier observation concerning the activation of JNK in insect fat body after LPS injection (Wojda et al., 2004). Nevertheless, in that case only JNK MAP pathway (and slightly ERK) was significantly activated in fat body, while in this report we show activation of both JNK and p38 pathways. This difference may be explained in at least two ways: first, LPS is a constituent of gram-negative bacteria, while Bacillus is gram-positive. Secondly, it is already known that the main activator of the immune system in insects is peptydoglycan rather than LPS (Kaneko et al., 2004; Charroux et al., 2009).

Phosphorylation of p38 in fat body was observed later, i.e. 30 minutes after infection, and reached the maximum at the 60-minute time-point. The increase in the level of this phospho-protein was not as sharp and transient as it was for JNK. This difference in the kinetics of p38 and JNK MAP kinase activation may suggest their different role in the regulation of the immune response. JNK MAP kinase may be involved in the early response, while p38 may control later events. JNK MAP kinase was reported to take place in wound healing and its role in the regulation of antimicrobial peptide synthesis is controversial. For example, in JNK MAPKKdeficient Drosophila melanogaster SL2 cells (mk.k4/hep), the expression of genes encoding cytoskeletal genes as well as a gene encoding attacin D after LPS treatment was abolished, while the expression of other genes encoding antimicrobial peptides did not change (Boutros et al., 2002). The possible role of this enzyme in the regulation of insect immunity was also reported by others and us (Wojda et al., 2004; Kallio et al., 2005). On the other 
hand, p38 MAP kinase pathway was reported to regulate attenuation of the immune response in Drosophila melanogaster (Han et al., 1998). The enhanced level of phosphorylated p38 in G. mellonella at later time-points after infection would be in agreement with that observation. However, taking into account that Bacillus thuringiensis is an enthomopathogen secreting many virulence factors, it is likely that some MAP kinases may be activated by the bacteria themselves, but some by their metabolites. We did not detect clear and reproducible activation of ERK MAP kinase in the fat bodies of the Bacillus-infected larvae, although we noticed the increase in the phosphorylation status of ERK in fat bodies of animals naturally infected with Beawveria bassiana (data not shown).

In hemocytes, a slight increase was observed for p38 and more pronounced for ERK, when bacteria were injected, in comparison to the injection of IPS alone. Similar to the case of the fat body, a stronger Bacillus-dependent increase in the amount of phospho-JNK MAP kinase (especially form a) was observed in the hemocytes of infected larvae, suggesting the role of this protein not only in abiotic stress response but also in fighting off pathogen infection. We also noticed that in hemocytes, aseptic injection itself caused a strong increase in the phospho-forms of all MAP kinases already 5 minutes after injection.

The investigation performed in our laboratory allowed a conclusion that $G$. mellonella exposed to heat-shock was more resistant to infection caused by the enthomopathogen. Additionally, we have recently published that $G$. mellonella pre-exposed to heat-shock showed enhanced immune response and their antimicrobial peptides were more resistant to degradation by intruder's proteases (Wojda et al., 2009, 2013). Considering also our previous finding, that the JNK MAP pathway is engaged in the appearance of defence activity in larval hemolymph (Wojda et al., 2004), we decided to compare the phosphorylation level of $\mathrm{JNK}$ in the fat bodies of larvae preexposed, or not to heat shock before they were infected with Bacillus thuringiensis. It appeared that the phosphorylation level of JNK was higher in the pre-shocked, infected animals in comparison to the insects which were only infected. Interestingly, this was not observed when G. mellonella were reared permanently at elevated temperature prior to infection (data not shown). It is likely that the JNK pathway may regulate not only immune response, but also the expression or activity of other stress proteins, protecting immune relevant proteins and peptides in $G$. mellonella hemolymph.

\section{REFERENCES}

Arrese EL, Soulages JL (2010) Insect fat body: energy, metabolism, and regulation. Annu Rev Entomol 55: 207-225.

Boutros M, Agaisse H, Perrimon N (2002) Sequential activation of signaling pathways during innate immune responses in Drosophila. Dev Cell 3: 711-722.

Boutros L, Chevet E, Metrakos P (2008) Mitogen-activated protein (MAP) kinase/MAP kinase phosphatase regulation: roles in cell growth, death, and cancer. Pharmacol Rev 6: 261-310.

Bradford M (1976) A rapid and sensitive method for the quantitation of microgram quantities of protein utilising the principle of proteindye binding. Anal Biochem 72: 248.

Charroux B, Rival T, Narbonne-Reveau K, Royet, J (2009) Bacterial detection by Drosophila peptidoglycan recognition proteins. Microb Infect 11: 631-636.

Glavis-Bloom J, Muhammed M, Mylonakis E (2012) Of model hosts and man: using Caenorbabditis elegans, Drosophila melanogaster and Galleria mellonella as model hosts for infectious disease research. Adv Exp Med Biol 710: 11-17.

Han ZS, Enslen H, Hu X, Meng X, Wu IH, Barrett T, Davis RJ, Ip YT (1998) A conserved p38 mitogen-activated protein kinase pathway regulates Drosophila immunity gene expression. Mol Cell Biol 18: 3527-3539.

Kallio J, Leinonen A, Ulvila J, Valanne S, Ezekowitz RA, Rämet M (2005) Functional analysis of immune response genes in Drosophila identifies JNK pathway as a regulator of antimicrobial peptide gene expression in S2 cells. Microb Infect 7: 811-819.

Kaneko T, Goldman WE, Mellroth P, Steiner H, Fukase K, Kusumoto S, Harley W, Fox A, Golenbock D, Silverman N (2004) Monomeric and polymeric gram-negative peptidoglycan but not purified LPS stimulate the Drosophila IMD pathway. Immunity 20: 637-649.

Khush RS, Leulier F, Lemaitre B (2001) Drosophila immunity: two paths to NF-кB. Trends in Immunol 22: 260-264.

Laemmli, U.K. (1970) Cleavage of structural proteins during the assembly of the head of bacteriophage T4. Nature 227: 680-685.

Lemaitre B, Kromer-Metzger E, Michaut L, Nicolas E, Meister M, Georgel P, Reichhart JM, Hoffmann JA (1995) A recessive mutation, immune deficiency (imd), defines two distinct control pathways in the Drosophila host defence. Proc Nat Acad of Sci USA 92: 9465-9469.

Lemaitre B, Nicolas E, Michaut L, Reichhart JM, Hoffmann JA (1996) The dorsoventral regulatory gene cassette spätzle/Toll/cactus controls the potent antifungal response in Drosophila adults. Cell 86: 973-983.

Li Y, Xiang Q, Zhang Q, Huang Y, Su Z (2012). Overview on the recent study of antimicrobial peptides: origins, functions, relative mechanisms and application. Peptides 37: 207-215.

Williams MJ (2007) Drosophila hemopoiesis and cellular immunity. J Immunol 178: 4711-4716.

Wojda I, Kowalski P, Jakubowicz T (2004) JNK MAP kinase is involved in the humoral immune response of the greater wax moth Galleria mellonella. Arch Insect Biochem Physiol 56: 143-54.

Wojda I, Kowalski P, Jakubowicz T (2009) Humoral immune response of Galleria mellonella larvae after infection by Beauveria bassiana under optimal and heat-shock conditions. J Insect Physiol 55: 525-531.

Wojda I (2010) Heat shock and the susceptibility of organisms to infection and osmotic challenge. Postepy Biochem 56: 83-94 (in Polish).

Wojda I (2012) MAP kinase pathways- their evolution and role in some neurodegenerative diseases. Postepy Biochem 58: 79-90 (in Polish).

Wojda I, Taszłow P (2013) Heat shock affects host-pathogen interaction in Galleria mellonella infected with Bacillus thuringiensis. J Insect Physiol 59: 894-905.

Zhang YL, Dong C (2005) MAP kinases in immune responses. Cell Mol Immunol. 2: 20-27. 